\title{
A HYBRIDIZED MONITOR RECEIVER FOR LOW BAND FM
}

\author{
ALAN T. BROWN \\ Dept Electronic Engineering, University of Pretoria, Republic of South Africa
}

\begin{abstract}
Certain aspects of the design and implementation of a hybridized monitor receiver are discussed. The receiver is realized in thick film technology. Screened capacitors as well as an adjustable inter-digitated thin film capacitor are employed in the design. Fine tuning of the crystal controlled local oscillator is achieved by abrasive trimming of monolithic chip capacitors. Modularization of the receiver lends itself towards simplified circuit construction and alignment.
\end{abstract}

\section{INTRODUCTION}

This paper describes the implementation of a portable monitor receiver for low band FM usage. Operation is on two or more pre-selectable fixed frequencies within $1 \mathrm{MHz}$ of each other in the band $30-50 \mathrm{MHz}$. Input sensitivity is $0.4 \mu \mathrm{V}$ for $12 \mathrm{~dB} \mathrm{~S} / \mathrm{N}, \pm 5 \mathrm{KHz}$ deviation. The monitor mode provides for the reception of an on/off modulated fixed frequency audio tone. Speech reception is however switch selectable when required.

Various approaches are possible for the implementation of such a receiver. In this case, reliability considerations coupled to miniaturization requirements favoured a hybrid implementation. The circuit is realized using thick film hybrid techniques.

Certain aspects of the design and implementation of the receiver will now be discussed.

\section{CIRCUIT DESCRIPTION}

A block diagram of the system is shown in Figure 1. A bandpass input filter precedes the RF gain stage which is coupled to the mixer. The local oscillator is crystal controlled and switchable between two or more crystals as required. The mixer output is fed via a monolithic crystal filter, providing the required channel selectivity to the IF amplifier and detector stages. In the monitor mode, an audio bandpass filter precedes the output stage. A switch bypasses this filter for speech reception when required.

The squelch stage controls an input monitoring function which periodically samples the input and locks the receiver on in the case of a present input signal. This provides a considerable power saving. Other monitoring functions include a battery indicator which

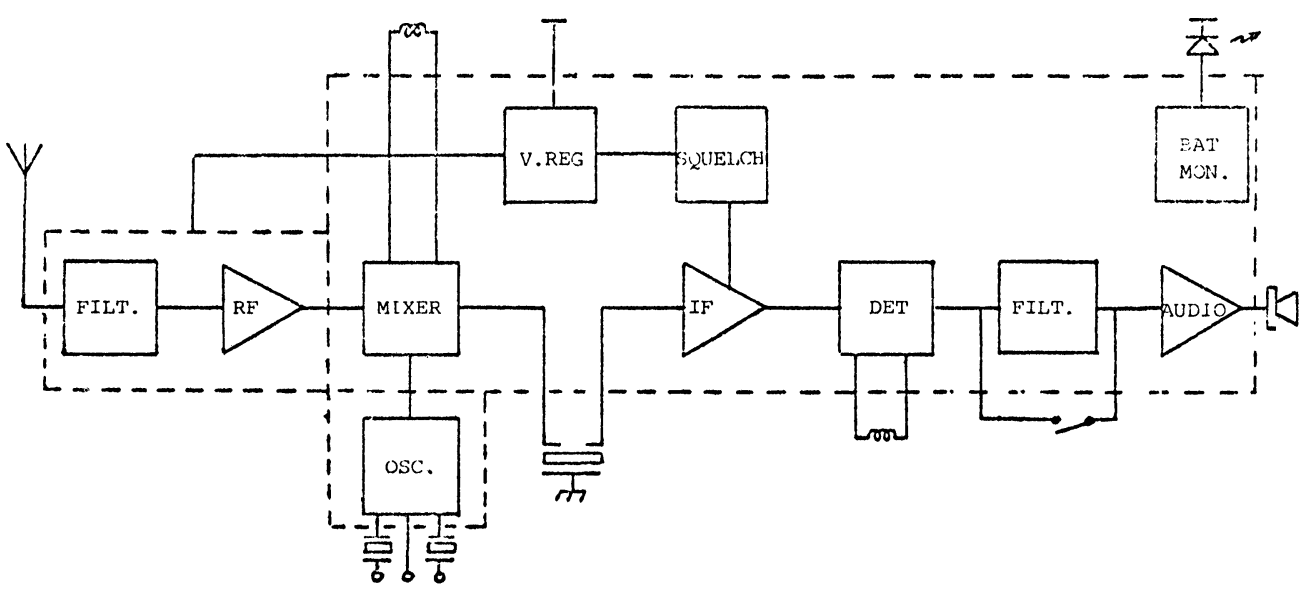

FIGURE 1 Block diagram of receiver. 
provides a visual indication of a low battery voltage. A voltage regulator ensures constant performance over a wide range, particularly with respect to squelch action.

\section{IMPLEMENTATION APPROACH}

The receiver circuitry is divided into three modules, each packaged in hermetically sealed, kovar platform style cans on a mother printed circuit board together with bulk components, e.g. oscillator crystals, crystal filter and IF inductors.

Modularization is as follows:

Substrate No 1: Input filter and RF amplifier (14 pin DIL package).

Substrate No 2: Crystal controlled local oscillator (14 pin DIL package).

Substrate No 3: Mixer, IF amplifier, detector and auxiliary circuits (25.4 $\mathrm{mm}$ sq. package).

This choice allows for:

1) RF screening of the sensitive input circuitry.

2) Minimization of radiation from the local oscillator.

3) Logical separation of the receiver functions allowing for a simplified alignment procedure.

The three substrates are screened simultaneously on a prescribed substrate. As the third substrate comprises two conductor layers, this afforded the opportunity of utilizing printed capacitors on substrates Nos 1 and 2 without additional screening steps. A trade-off was required between dielectric compositions suitable for multilayer circuitry as opposed to those more suited for screened capacitors. The composition chosen was DU PONT 9429. DU PONT 9841 is more appropriate as a capacitor dielectric but does not provide optimum via resolution required for the multilayer substrate, due to its lower viscosity. ${ }^{3}$ An investigation of the 9429 composition as a capacitor dielectric yielded acceptable results. These are summarized in Figure 2.

The bar graph shows the results of measurements on a series of screened capacitors ranging in area from 1 to $16 \mathrm{~mm}^{2}$. Normalized capacitance spread and standard deviation are shown. A double screening of 9429 composition was used, the substrates being fired after each screening. Final fired thickness was $30 \mu \mathrm{m}$.

As indicated, capacitance spread remains below $\pm 10 \%$ with a standard deviation of less than $5 \%$. A capacitive density of $3.6 \mathrm{pF} / \mathrm{mm}^{2}$ was obtained. Voltage coefficient measured between 0 and $100 \mathrm{~V}$ is negligible and the temperature coefficient measured

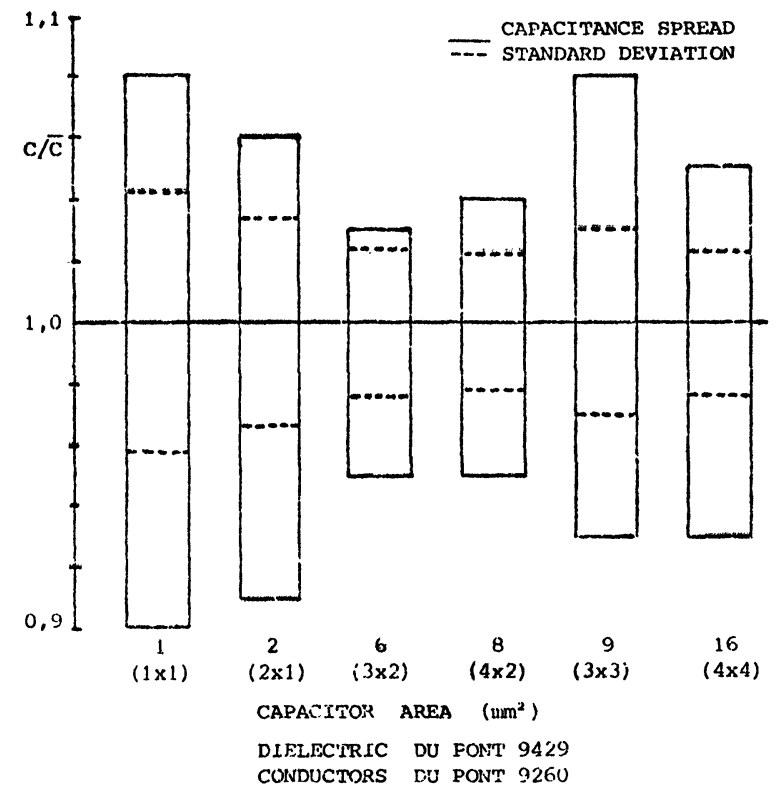

FIGURE 2 Screened capacitor results.

over the range $-30^{\circ} \mathrm{C}$ to $+100^{\circ} \mathrm{C}$ is $95 \mathrm{ppm} /{ }^{\circ} \mathrm{C}$. This represents less than $1 \%$ change in capacitance over a temperature range of $100^{\circ} \mathrm{C}$.

The screened capacitors utilizing this dielectric are used essentially in tuned circuits. The maximum spread of $\pm 10 \%$ is compensated for by inductor tuning. The $1 \%$ capacitance change with temperature can be tolerated in the circuit.

A further decision to screen decoupling capacitors on substrates Nos 1 and 2 simplified the construction of these modules. DU PONT 8289 composition was chosen for this purpose. Capacitors implemented with this composition typically exhibit a temperature change of $+10,-20 \%$ over the range $-30^{\circ} \mathrm{C}$ to $+80^{\circ} \mathrm{C}$. This, however, does not significantly affect circuit behaviour.

\section{SUBSTRATE NO. 1 - RF AMPLIFIER AND INPUT FILTER}

This substrate houses the input filter, dual gate MOSFET stage and output matching network (Figure 3).

The filter is a twin coupled resonator. Adjustment of the filter requires the tuning of both resonators $\left(T_{1}, C_{1}\right.$ and $L_{2}, C_{2}$ ) to the required frequency as well as adjustment of the capacitor, $C_{m}$, to provide the required degree of coupling. Three adjustable elements 


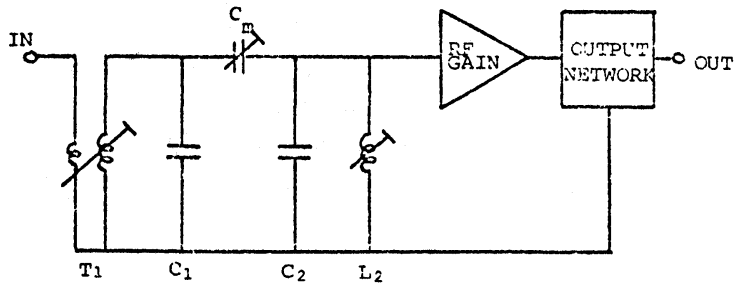

FIGURE 3 Details of input amplifier.

were thus required, all of which were to be mounted inside the package.

Capacitors $C_{1}$ and $C_{2}$ are screened onto the substrate and toroidal inductors $\left(T_{1}\right.$ and $\left.L_{2}\right)$ are adjusted to effect tuning of the resonators. An analysis of the filter shows that for equal valued resonator capacitors $\left(C_{1}=C_{2}\right)$ the coupling capacitor required for a maximally flat response is:

$$
C_{m}=\frac{C}{\sqrt{ } 2 Q_{F}}
$$

where $C=C_{1}=C_{2}$

$Q_{F}=$ Filter $Q$

$=$ Centre frequency/filter bandwidth

Requirements for the receiver were a $Q_{F}$ of 25 . The screened capacitors measured nominally $45 \mathrm{pF}$. A coupling capacitor of the order of $1.8 \mathrm{pF}$ was thus required.

Conventional capacitors proved to be inconvenient due to their size and attachment methods. Miniature devices are also as a rule not cost effective. Available data concerning interdigitated capacitors, ${ }^{1}$ indicates that capacitive densities of $0.25 \mathrm{pF} / \mathrm{mm}^{2}$ are typical for electrode width and spacing of $0.1 \mathrm{~mm}$.

As line resolution of $0.1 \mathrm{~mm}$ exceeds the current capabilities of a high yield thick film production run, a thin film interdigitated device was manufactured with outer dimensions of $3 \times 3 \mathrm{~mm}$, thereby providing a theoretical capacitance of $2.25 \mathrm{pF}$. A modification of the basic device enabled the capacitance to be adjusted by the successive connection of electrodes, using $25 \mu \mathrm{m}$ gold wire, thermocompression bonding. The capacitor is shown in Figures 4 and 5 with results achieved shown in Figure 6.

The tuning procedure of the filter involves the initial mounting of the thin film capacitor with minimum capacitance, i.e. no electrodes connected. Resonator inductors are now adjusted for the appropriate frequency range. Electrodes on the thin film capacitor are now successively bonded in to provide the coupling required. Appropriate connections to the substrate allow for the tuning of the filter before the active device

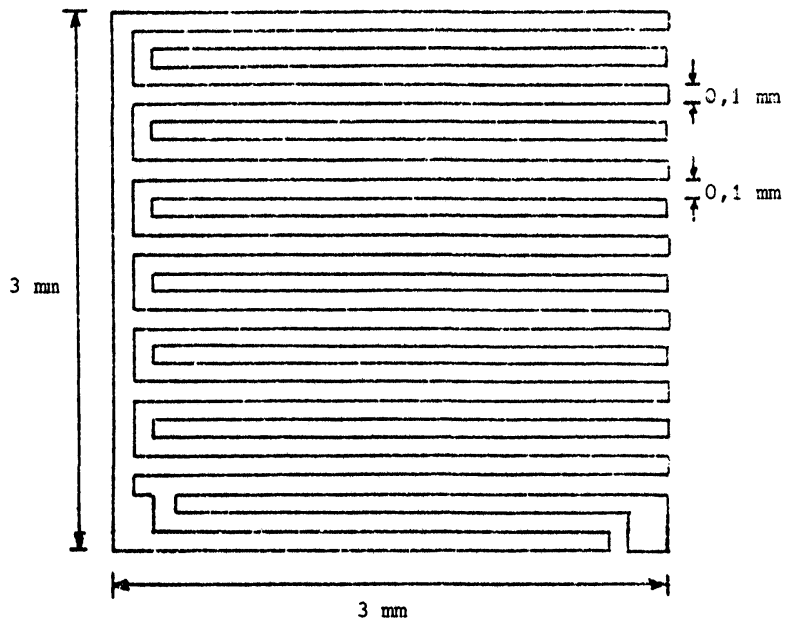

FIGURE 4 Details of thin film capacitor.

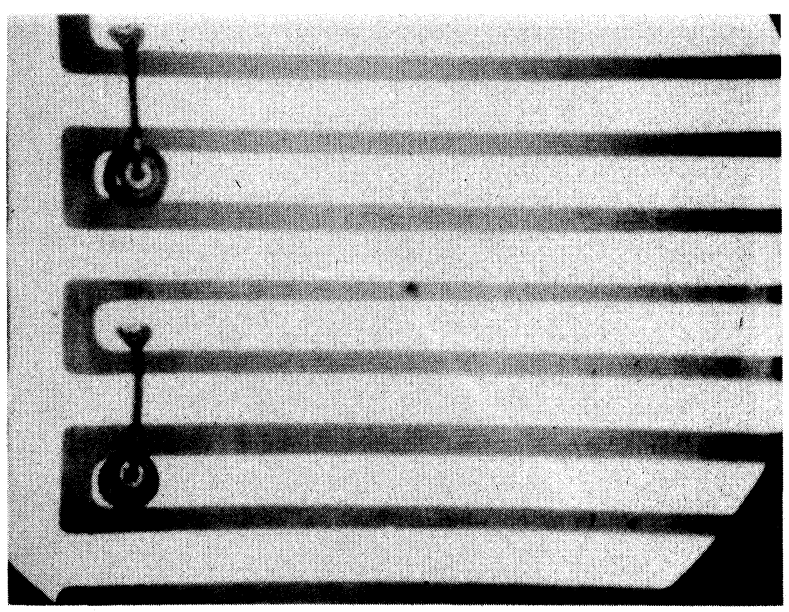

FIGURE 5 Bonding of electrodes on thin film capacitor.

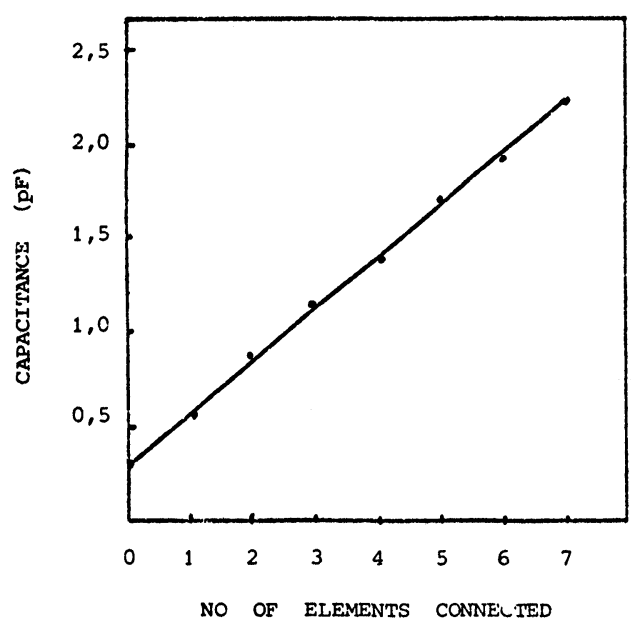

FIGURE 6 Results achieved with thin film capacitor. 


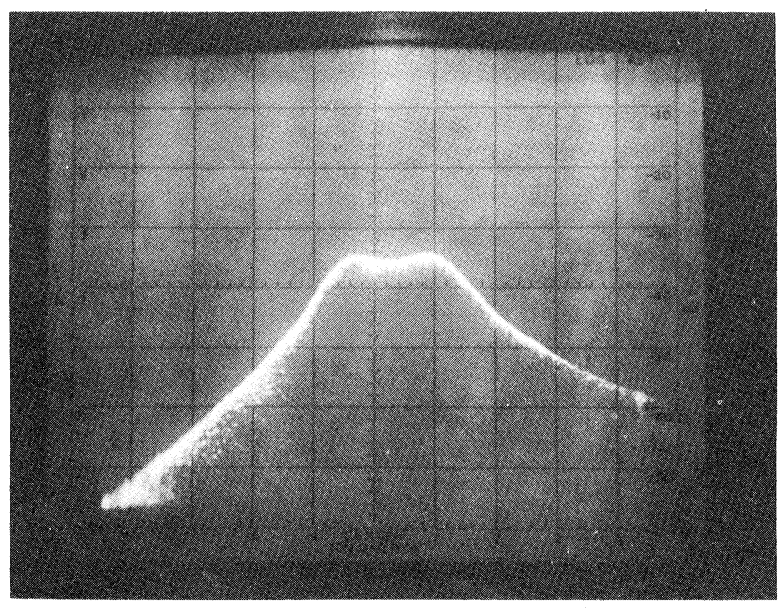

FIGURE 7a Filter response corresponding to 6 bonded electrodes.

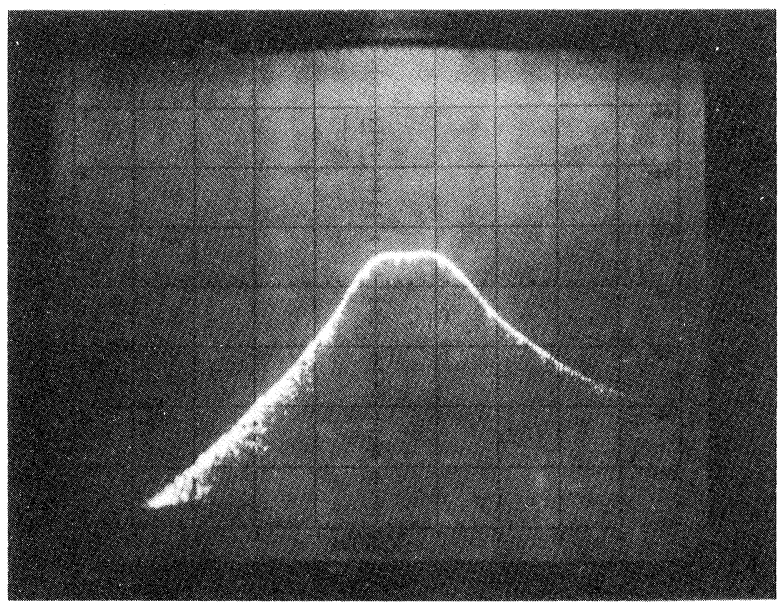

FIGURE $7 \mathrm{~b}$ Filter response corresponding to 5 bonded electrodes.

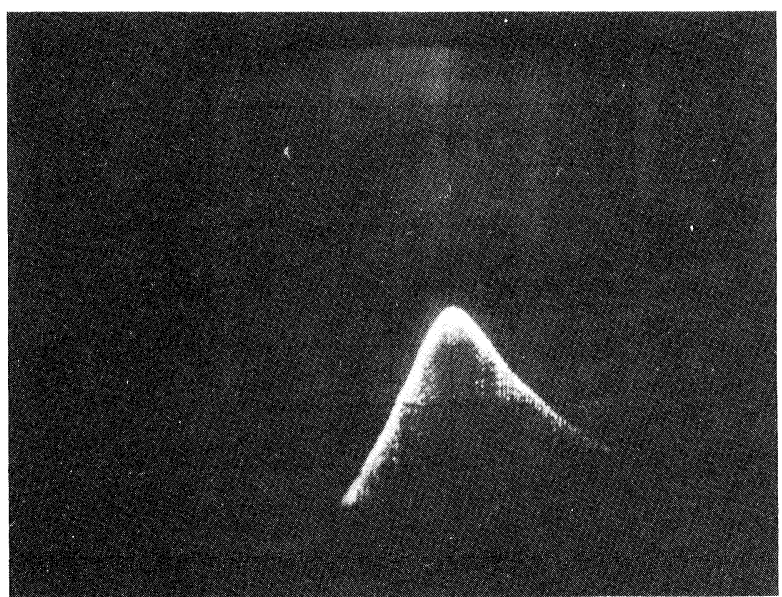

FIGURE 7c Filter response corresponding to 1 bonded electrode. is mounted. The filter response, corresponding to the number of elements connected, is shown in Figure 7.

The remainder of the circuit consists of the MOSFET device in chip form and output matching network. Screened capacitors are used throughout with tuning once again relying on toroidal inductor adjustment. The completed module is shown in Figure 8 .

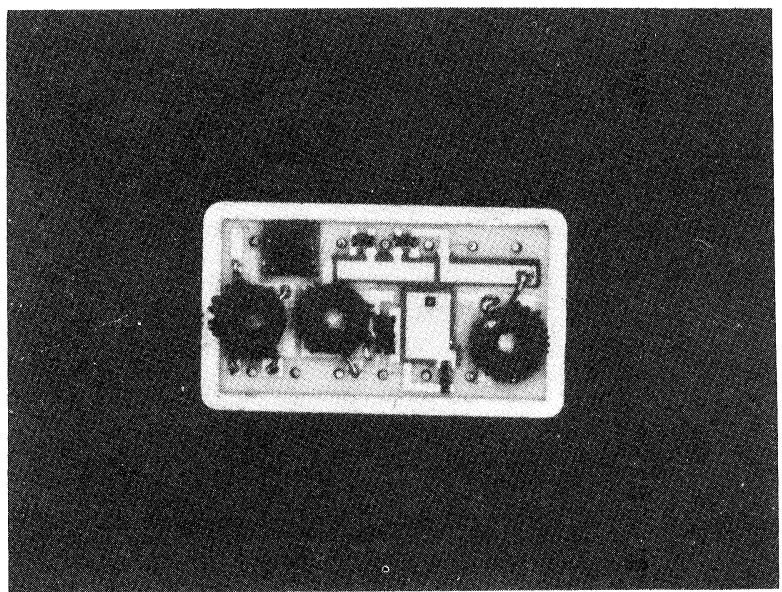

FIGURE 8 RF amplifier module. Package dimensions $22 \times 12.5 \mathrm{~mm}$.

\section{SUBSTRATE NO. 2 - LOCAL OSCILLATOR}

The local oscillator provides for operation on two preselectable crystal controlled frequencies and an auxiliary function allowing for a third fixed frequency or else further expansion of the available channels.

Fine tuning of the crystal frequencies is achieved by abrasive trimming of series connected monolithic chip capacitors. ${ }^{2}$ This is performed actively with feedback from a frequency counter controlling the trimming action. Trimming is carried out on the completed circuit, each module being matched to its specific crystals. The active device used here is SOT 23 packaged rather than a chip device.

All other capacitors are screened onto the substrate. Tuning is by toroidal inductor adjustment. Three of the screened capacitors are not used in tuned circuits. Two of these determine the starting criteria of the oscillator. This is largely capacitance ratio dependant which is generally more closely controlled than absolute values. The third capacitor provides coupling to the high input impedance mixer stage. Slight variations in the value of this capacitor will have minimum effect. The completed module is shown in Figure 9. 


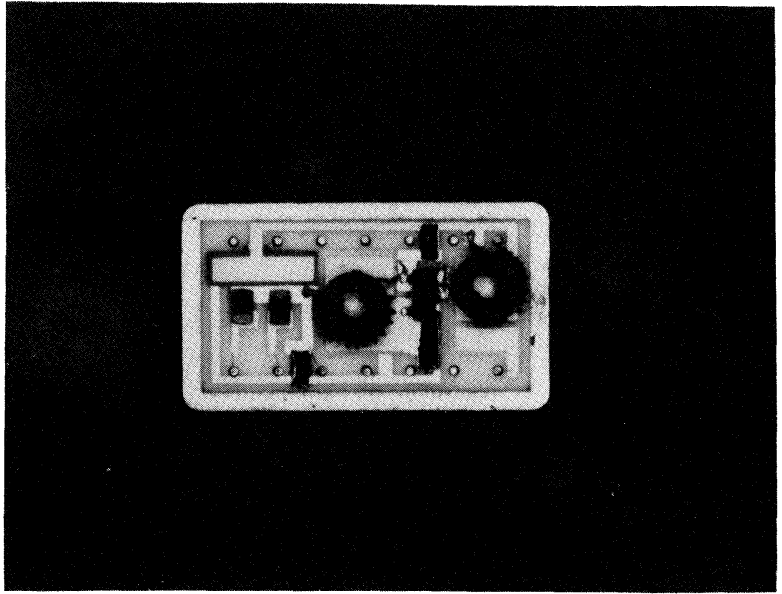

FIGURE 9 Oscillator module. Package dimensions $22 \times 12.5 \mathrm{~mm}$.

\section{SUBSTRATE NO. 3 - MIXER/DETECTOR AND AUXILIARY CIRCUITS}

Because of its relatively high circuit density, this substrate is implemented with double layer thick film techniques. Where possible, circuitry utilizes non-critical resistor ratios rather than absolute values. Few resistors thus require trimming, an important consideration for high density circuitry.
Alignment of the circuit entails only tuning of the quadrature detector and mixer tuned circuits. In both cases, this involves the adjustment of an externally mounted inductor. Access is provided via the package to critical points in the circuit allowing for a simplified alignment procedure as well as the monitoring of signals at key points.

\section{CONCLUSION}

1) Utilization of thick film technology has effected a space-efficient receiver design.

2) This technique has allowed for logical modularization of the circuitry, affording convenient alignment procedures.

3) An adjustable thin film interdigitated capacitor has been sucessfully utilized as the tuning element of the input filter.

4) Screened capacitors have been used to good effect on two of the modules.

\section{REFERENCES}

1. Holmes, P. J. Handbook of Thick Film Technology, pp. 207-211.

2. Weightman, G. Abrasive Trimming - The Important Forgotten Tool. Proceedings of the 1977 International Microelectronics Conference. pp. 25-28.

3. Du Pont, Product Literature, 9429 and 9841 data sheets. 

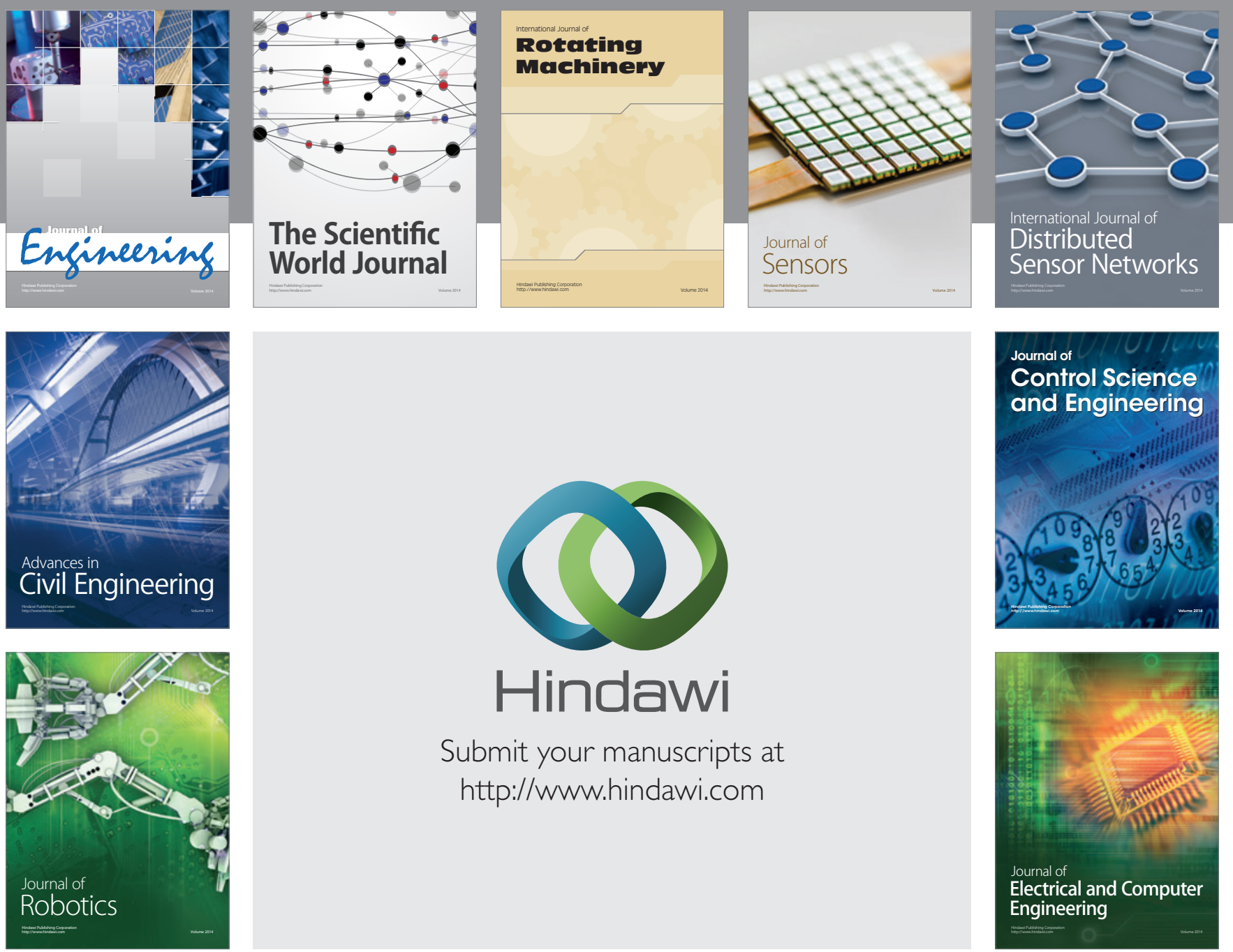

Submit your manuscripts at

http://www.hindawi.com
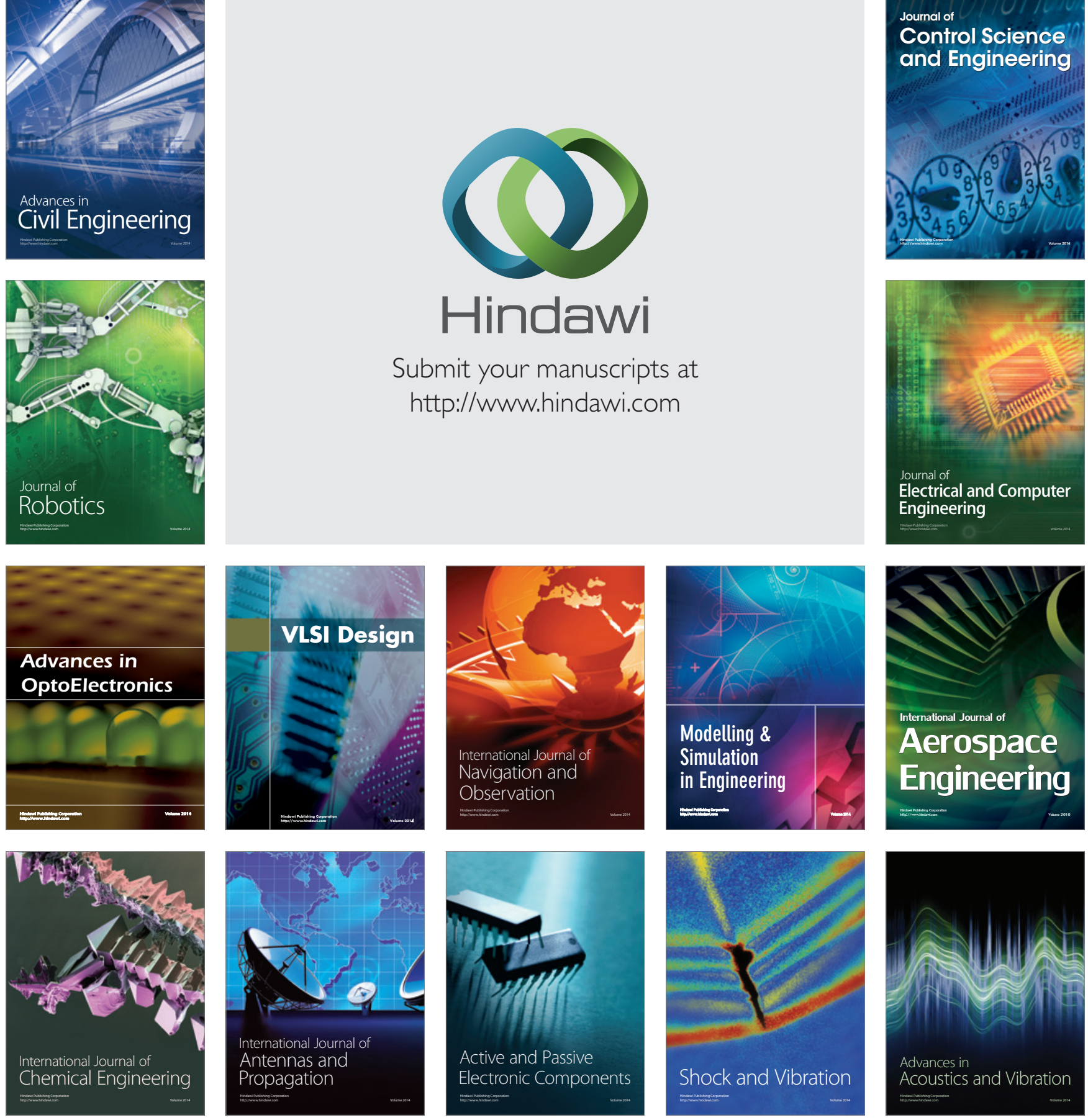